ARTICLE

https://doi.org/10.1038/s41467-019-13391-z

\title{
Imaging and writing magnetic domains in the non-collinear antiferromagnet $\mathrm{Mn}_{3} \mathrm{Sn}$
}

Helena Reichlova*, Tomas Janda2 ${ }^{1 \star}$ Joao Godinho 2,3, Anastasios Markou (1) 4, Dominik Kriegner (1) 3,4, Richard Schlitz ${ }^{1}$, Jakub Zelezny ${ }^{3}$, Zbynek Soban ${ }^{3}$, Mauricio Bejarano ${ }^{5}$, Helmut Schultheiss ${ }^{5}$, Petr Nemec ${ }^{2}$, Tomas Jungwirth ${ }^{3,6}$, Claudia Felser (i) ${ }^{4}$, Joerg Wunderlich ${ }^{3,7}$ \& Sebastian T.B. Goennenwein (1) ${ }^{1}$

Non-collinear antiferromagnets are revealing many unexpected phenomena and they became crucial for the field of antiferromagnetic spintronics. To visualize and prepare a well-defined domain structure is of key importance. The spatial magnetic contrast, however, remains extraordinarily difficult to be observed experimentally. Here, we demonstrate a magnetic imaging technique based on a laser induced local thermal gradient combined with detection of the anomalous Nernst effect. We employ this method in one the most actively studied representatives of this class of materials $-\mathrm{Mn}_{3} \mathrm{Sn}$. We demonstrate that the observed contrast is of magnetic origin. We further show an algorithm to prepare a well-defined domain pattern at room temperature based on heat assisted recording principle. Our study opens up a prospect to study spintronics phenomena in non-collinear antiferromagnets with spatial resolution.

\footnotetext{
${ }^{1}$ Institut für Festkörper- und Materialphysik and Würzburg-Dresden Cluster of Excellence ct.qmat, Technische Universität Dresden, 01062 Dresden, Germany

${ }^{2}$ Faculty of Mathematics and Physics, Charles University, Ke Karlovu 3, 12116 Prague 2, Czech Republic. ${ }^{3}$ Institute of Physics, Czech Academy of Sciences, Cukrovarnická 10, 16200 Praha 6, Czech Republic. ${ }^{4}$ Max Planck Institute for Chemical Physics of Solids, Nöthnitzer Straße 40, 01187 Dresden, Germany.

${ }^{5}$ Helmholtz-Zentrum Dresden-Rossendorf, Institute of Ion Beam Physics and Materials Research, Bautzner Landstraße 400, 01328 Dresden, Germany.

${ }^{6}$ School of Physics and Astronomy, University of Nottingham, NG7 2RD Nottingham, UK. ${ }^{7}$ Hitachi Cambridge Laboratory, Cambridge CB3 OHE, UK.

*email: helena.reichlova@tu-dresden.de
} 
arnessing the unique properties of non-collinear antiferromagnets (AFMs) will be essential for exploiting the full potential of antiferromagnetic spintronics ${ }^{1,2}$. Indeed, many of the effects enabling ferromagnetic spintronic devices have a corresponding counterpart in materials with non-collinear spin structure ${ }^{3-8}$. In addition, phenomena, such as the magnetic spin Hall effect ${ }^{9}$ or the chiral anomaly ${ }^{10}$ were experimentally observed in non-collinear AFMs, and the presence of the equivalent to the ferromagnetic spin transfer torque via spin polarized currents was theoretically predicted ${ }^{11}$. In spite of these developments, an interpretation of the rich physical phenomena observed in non-collinear antiferromagnets is challenging, since the microscopic spin arrangement, the magnetic domain distribution, and the domain orientations have proven notoriously difficult to access experimentally.

This is all the more problematic, as imaging and writing magnetic domains is of central importance for applications. Successful imaging is a basic requirement to experimentally confirm the spin transfer torque acting on non-collinear domain walls and therefore of eminent interest. Surface magnetic character of a non-collinear antiferromagnetic single crystal was probed via magneto-optical Kerr effect (MOKE) ${ }^{3}$. MOKE is, however, mostly surface sensitive and together with several other restrictions (details in Supplementary Note 1) MOKE might be of limited use for certain spatially resolved studies. Here we report, that the local magnetic structure of the non-collinear AFM $\mathrm{Mn}_{3} \mathrm{Sn}$ films can be imaged by scanning thermal gradient microscopy (STGM $)^{12-14}$. The technique is based on scanning a laser spot over the sample's surface, and recording the ensuing thermo-voltage. We image the magnetic structure at a series of different temperatures and show that at room temperature, the domain structure is not affected by the application of moderate magnetic fields. In addition to imaging, we establish a scheme for heat-assisted magnetic recording, using local laser heating in combination with magnetic fields to intentionally write domain patterns into the antiferromagnet.

\section{Results}

The antiferromagnetic semimetal $\mathbf{M n}_{3} \mathrm{Sn}$. The antiferromagnetic semimetal $\mathrm{Mn}_{3} \mathrm{Sn}$ is a prime representative of materials with a triangular spin structure and it is very actively discussed in the context of Weyl physics ${ }^{4,10,15-18}$. The material is of particular interest due to the topology of its electronic bands with strong Berry curvature contributions to anomalous magneto-transport ${ }^{19,6}$. It has hexagonal structure with $\mathrm{P6}_{3} / \mathrm{mmc}$ space group with the magnetic moments residing in a c-plane kagome lattice (Fig. 1a). Fig. 1 shows the spin configuration experimentally confirmed in bulk $\mathrm{Mn}_{3} \mathrm{Sn}^{20}$, but several spin arrangements in the c-plane are discussed as energetically equivalent ${ }^{21-24}$. Considering that our films exhibit the same Néel temperature $T_{\mathrm{N}}=420 \mathrm{~K}$ as bulk $\mathrm{Mn}_{3} \mathrm{Sn}$, we expect that they also have an identical spin structure. The anisotropy of the three magnetic sublattices partly cancels out ${ }^{20}$ which allows for the experimental manipulation of the antiferromagnetic order.

The $\mathrm{Mn}_{3} \mathrm{Sn}$ epitaxial thin films studied here were prepared by ultra-high vacuum (UHV) sputtering ${ }^{25}$ (details on fabrication and characterization are compiled in the Methods, Supplementary Notes 2, 3 and Supplementary Figs. 1, 2). The films are oriented, such that the [001] crystal direction (c-axis) points outof-plane and $5 \mu \mathrm{m}$ wide Hall bars patterned by optical lithography are typically oriented along the [100] crystal direction of $\mathrm{Mn}_{3} \mathrm{Sn}$. A typical device and the experimental geometry are shown in Fig. 1c, d. The sample is placed in an optical cryostat with the magnetic field applied along the $\mathrm{x}$-direction (perpendicular to the Hall bar). We scan a focused red laser $(\lambda=800 \mathrm{~nm}, P$
$=10 \mathrm{~mW}$ ) across the Hall bar and record the ensuing thermovoltage along the y-direction (Fig. 1c). As evident from Fig. 1e, the spatially resolved thermo-voltage response clearly reveals spatial contrast.

The anomalous Nernst effect. We discuss the origin of the signal in our thermal gradient microscopy. From a symmetry point of view, the anomalous Hall effect (AHE) is equivalent to a timereversal odd axial vector $\mathbf{g}^{26,27}$ such that anomalous Hall current $j_{\mathrm{AHE}}=\mathbf{g} \times \mathbf{E}$ where $\mathbf{E}$ is the electric field. The anomalous magneto transport plane (the plane in which the electric fields or currents are applied and recorded) is then perpendicular to $\mathrm{g}$. Considering the Mott relation the symmetry of the anomalous Nernst effect (ANE) and AHE is identical ${ }^{28}$ with the applied electric field $\mathbf{E}$ replaced by a thermal gradient $\nabla T$, therefore, $V_{\mathrm{ANE}} \sim \nabla T \times \mathbf{g}$. The orientation of the vector $\mathbf{g}$ is determined by the non-collinear structure, as illustrated in Fig. 1b for two opposite domains. Note that the symmetry properties of the vector $\mathbf{g}$ also result in a tendency of the material to develop a net magnetic moment along the $\mathbf{g}$ vector. This net moment is, however, very weak $\left(\sim 0.002 \mu_{\mathrm{B}} /\right.$ f. $\mathrm{u}^{5}$ ) and is not the source of the strong anomalous transport coefficients 5,27 in $\mathrm{Mn}_{3} \mathrm{Sn}$.

As demonstrated ${ }^{4}$ (see also Supplementary Note 4 and Supplementary Fig. 3), the anomalous Nernst response of $\mathrm{Mn}_{3} \mathrm{Sn}$ is strongly anisotropic. No anomalous Nernst voltage $V_{\mathrm{ANE}}$ is expected when the thermal gradient $\nabla T$ is applied in the c-plane ${ }^{4}$, while $V_{\mathrm{ANE}}$ in the direction perpendicular to the $\mathbf{g}$ vector is expected when $\nabla T$ is applied along the c-axis. In our sample, laser generated heat drains into the substrate, the in-plane components (in c-plane) compensate each other and the remaining thermal gradient is along the $\mathrm{z}$-direction (c-axis). Magnetic field is applied along the $\mathrm{x}$-direction and we detect $V_{\mathrm{ANE}}$ along the y-direction. Thus, the thermo-voltage signal is determined by the projection of the $\mathbf{g}$ vector onto the $\mathrm{x}$-direction. The different magnitude and sign of $V_{\text {ANE }}$ observed depending on the position $(\mathrm{x}, \mathrm{y})$ of the laser spot on the sample thus reflects the corresponding local orientation of the vector $\mathbf{g}$ in the irradiated area. Note that the laser beam is focused to a diameter of $1.5 \mu \mathrm{m}$, such that $V_{\mathrm{ANE}}$ reflects the average of possibly different g orientations or domains present within the illuminated spot. The measured voltage is therefore proportional to the net (average) component of the $\mathbf{g}$ vectors perpendicular to the detection direction. The resolution of the STGM is discussed in more detail in the Supplementary Note 5. Remarkably, we observe very different $V_{\text {ANE }}$ patterns depending on the sample history. As discussed below, magnetic domains can be intentionally written into the $\mathrm{Mn}_{3} \mathrm{Sn}$ using local heating. The domain pattern shown in Fig. 1e is the result of such a process. On the other hand, upon cooling the sample from $T>T_{\mathrm{N}}$ in zero magnetic field, domains are randomly populated resulting in more complex magnetic spatial contrast, as can be seen for example in Fig. 2a, b. Several Hall bars with different orientation with respect to the [100] direction of $\mathrm{Mn}_{3} \mathrm{Sn}$ were measured showing similar results.

Scanning thermal gradient microscopy. In this section, we demonstrate that the $V_{\mathrm{ANE}}$ signal is indeed of magnetic origin, and that it can be reversed by an external magnetic field. It is important to note that the magnetic field required for the reversal of spins in $\mathrm{Mn}_{3} \mathrm{Sn}$ thin films is higher compared to bulk $\mathrm{Mn}_{3} \mathrm{Sn}$ crystals ${ }^{29,30}$. Therefore, in our thin film samples, the spin structure cannot be reversed by $0.5 \mathrm{~T}$ (the experimentally available field) at temperatures below $300 \mathrm{~K}$, as can be seen in Fig. 2a, b. At $375 \mathrm{~K}$ a small variation of the measured $V_{\mathrm{ANE}}$ signal depending on the polarity of the magnetic field can be seen (Supplementary 
a

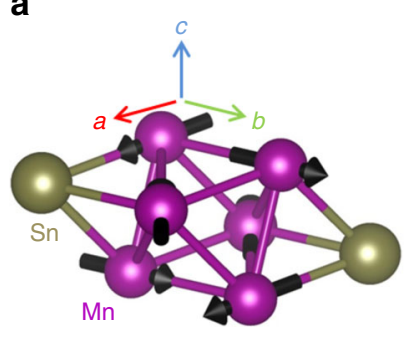

C

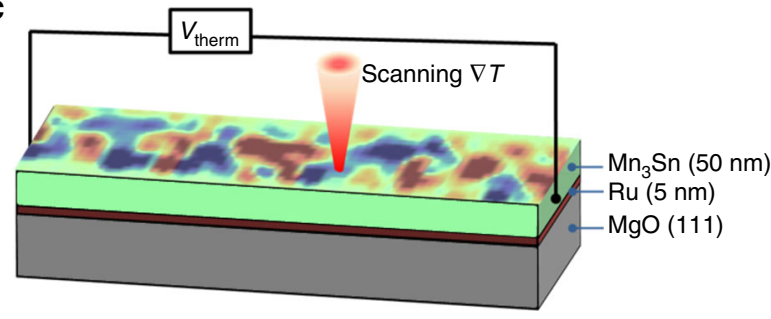

b
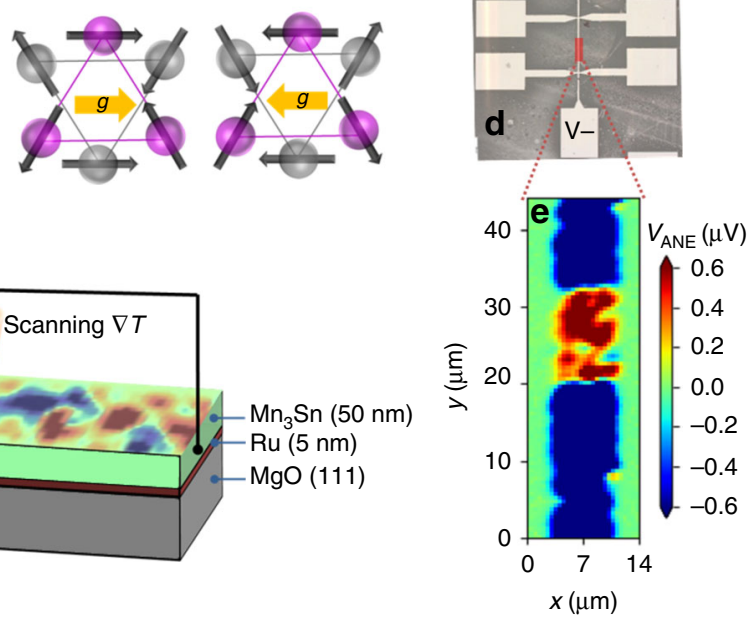

Fig. 1 Crystal structure and experimental setup. a $M n_{3} S n$ has hexagonal structure with $P \sigma_{3} / m m c$ space group. The magnetic moments (spins) are residing in the c-plane. $\mathbf{b}$ Two opposite magnetic domains with the corresponding $\mathbf{g}$ vectors (yellow arrows). c Schematics of STGM. Mn ${ }_{3} \mathrm{Sn}$ films are grown on $\mathrm{MgO}$ (111) substrates with a $5 \mathrm{~nm}$ Ru underlayer. The laser beam is scanned over the sample surface and the resulting local thermo-voltage sign and magnitude reflects the local magnetic properties. $\mathbf{d}$ Microscope image of a typical device and the experimental geometry. e Image of the spatially resolved thermo-voltage recorded in the sample (in the part highlighted as a red rectangle in (d)). The thermo-voltage signal reveals clear magnetic contrast. The experiment was performed at $300 \mathrm{~K}$, with a laser power of $10 \mathrm{~mW}$, and in zero external magnetic field.
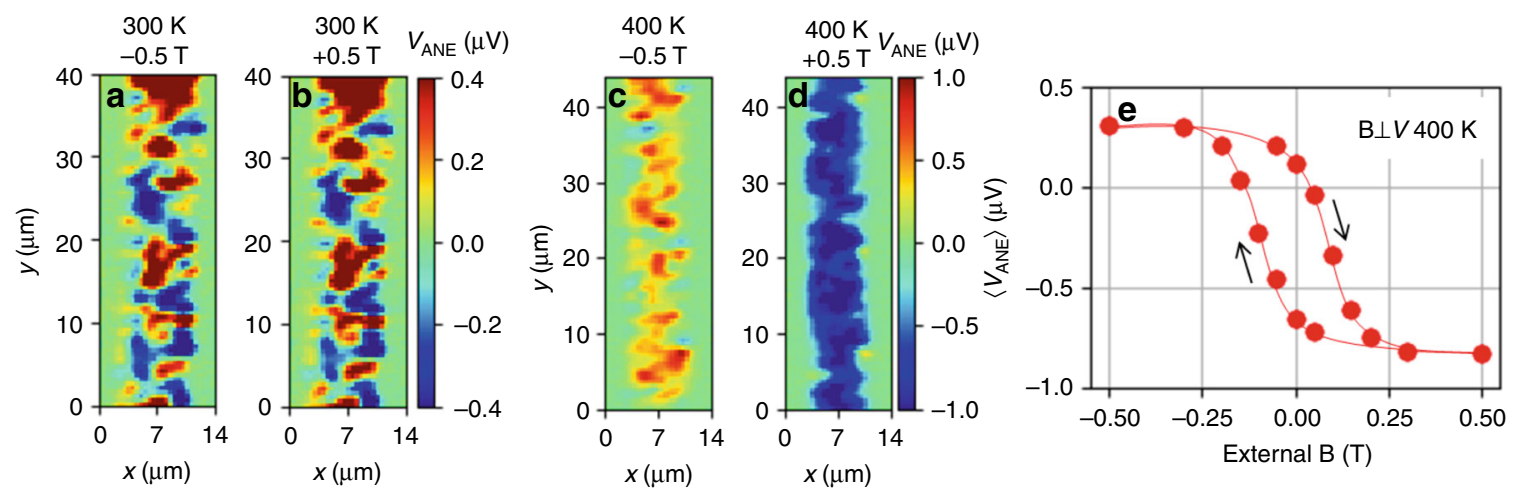

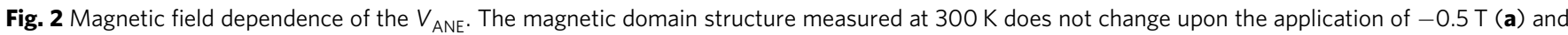
$+0.5 \mathrm{~T}$ (b) magnetic field. However, at $400 \mathrm{~K}$, the domain structure can be qualitatively altered by $-0.5 \mathrm{~T}$ (c) and $+0.5 \mathrm{~T}$ (d). e Shows the average $\left\langle V_{\mathrm{ANE}}\right\rangle$ within the scanned region as a function of the magnetic field at $400 \mathrm{~K}$. All scans were taken with a laser power of $10 \mathrm{~mW}$. The red line is a guide for the eye.

Note 6, Supplementary Fig. 4) corresponding to a reorientation of domains with the weakest coercivity. In contrast, increasing the sample temperature to $400 \mathrm{~K}$ and then applying a magnetic field allows us to completely alter the domain pattern, as shown in Fig. $2 c$, d. At $400 \mathrm{~K}$, the majority of domains is following the external magnetic field. A magnetic field of $-0.5 \mathrm{~T}$ yields a positive STGM map (red color) across the entire region scanned with the laser spot (Fig. 2c), while for $+0.5 \mathrm{~T}$, the STGM map turns negative (blue color) (Fig. 2d). This shows that at $400 \mathrm{~K}$, which is close to the Néel temperature $T_{\mathrm{N}}=420 \mathrm{~K}$, a magnetic field of $0.5 \mathrm{~T}$ suffices to align the $\mathbf{g}$ vector along the field direction in the entire sample. At the same time the net magnetic moment detected by SQUID magnetometry (see Supplementary Fig. 2) remains unchanged between $300 \mathrm{~K}$ and $400 \mathrm{~K}$, such that ferromagnet-like phases appearing at higher temperatures can be excluded. To further study the impact of the magnetic field on the magnetic domain pattern, we recorded STGM maps for several different magnetic field values in a field sweep. As detailed in the Supplementary Note 7, a complex reversal behavior with multiple domains is observed (for individual maps at each magnetic field see Supplementary Fig. 5). In Fig. 2e, we plot the voltage $\left\langle V_{\mathrm{ANE}}\right\rangle$ averaged over the whole scanned area as a function of the field strength, and find a global magnetic hysteresis curve with a clear saturation, coercivity and remanence. Since we observe a sign reversal in $\left\langle V_{\mathrm{ANE}}\right\rangle$ as a function of the magnetic field, the signal is odd under spin reversal and, therefore, the main contribution has the Nernst symmetry. The magneto-thermo-voltage clearly cannot be explained by an ordinary Nernst effect, which is linear in magnetic field and does not show hysteresis. Instead, the observed $V_{\mathrm{ANE}}$ must be connected to the magnetic order parameter of the antiferromagnet. Moreover, when applying the magnetic field parallel to the voltage detection direction, $\left\langle V_{\mathrm{ANE}}\right\rangle$ shows no remanence or saturation at $400 \mathrm{~K}$ (Supplementary Note 4, Supplementary Fig. 3). This supports the notion that the component of $\mathbf{g}$ perpendicular to the voltage detection determines $V_{\mathrm{ANE}}$.

An additional confirmation that the spatial contrast of STGM maps is governed by the antiferromagnetic order in $\mathrm{Mn}_{3} \mathrm{Sn}$ is evident from the evolution of the STGM signal with temperature. 

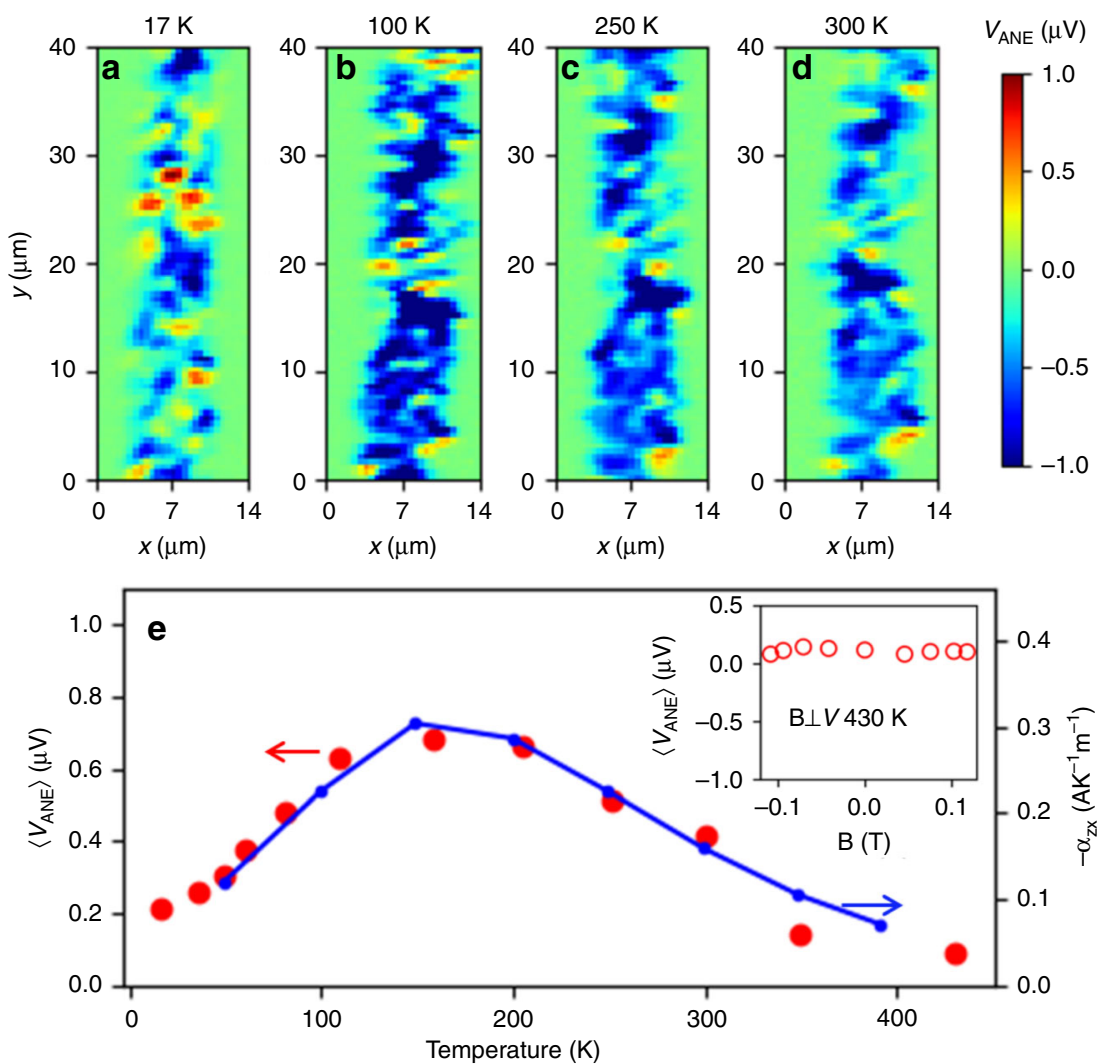

Fig. 3 Temperature dependency of the measured signal. $V_{\text {ANE }}$ changes with the sample temperature, as evident from the STGM maps at $17 \mathrm{~K}$ (a), $100 \mathrm{~K}$ (b), $250 \mathrm{~K}(\mathbf{c})$, and $300 \mathrm{~K}(\mathbf{d})$. All scans were taken with a laser power of $10 \mathrm{~mW}$. e The average $\left\langle V_{\mathrm{ANE}}\right\rangle(\mathrm{T})$ (red circles) in our thin film exhibits the same temperature dependence as the anomalous Nernst effect reported in the bulk (blue line, data reproduced from ref. ${ }^{4}$ ). Inset: Averaged voltage $\left\langle V_{\mathrm{ANE}}\right\rangle$ as a function of external magnetic field (equivalent to the experiment in Fig. 2e) at $430 \mathrm{~K}$ (above the Néel temperature of $\mathrm{Mn}_{3} \mathrm{Sn}$ ).

Fig. 3 shows $V_{\mathrm{ANE}}$ scans taken at different $T$ in the range of 17-430 K (for more temperatures see Supplementary Fig. 6 and Supplementary Note 8). The sample was first cooled to $17 \mathrm{~K}$ in zero magnetic field and then warmed up step by step to higher temperatures. No magnetic field was applied during this experiment, such that also data at $400 \mathrm{~K}$ exhibit a lower voltage $\left\langle V_{\mathrm{ANE}}\right\rangle$ compared to the polarized state (Fig. 2). We find a subtle but robust variation with $T$. The net amplitude $\left\langle V_{\mathrm{ANE}}\right\rangle$ plotted in Fig. 3e peaks in the vicinity of $150 \mathrm{~K}$, in excellent agreement with the temperature dependence of the anomalous Nernst response reported in bulk $\mathrm{Mn}_{3} \mathrm{Sn}$ crystals ${ }^{4}$ which is reproduced in Fig. 3e (blue line). Above $T_{\mathrm{N}}=420 \mathrm{~K}$, the thermo-voltage signal vanishes. Indeed, the magnetic field dependent experiments performed at $430 \mathrm{~K}$, depicted in the inset of Fig. 3e, show that $\left\langle V_{\mathrm{ANE}}\right\rangle$ is zero, with no signs of remanence or saturation. Note that we did not detect any evidence for a spin glass phase in our samples below $50 \mathrm{~K}^{5}$. Here again, the different magnetic anisotropy of thin films might affect the presence of the spin glass phase ${ }^{31,32}$.

The STGM does not allow for a direct evaluation of the anomalous Nernst coefficient. The reason is that, unlike in the case of a conventional in-plane thermal gradient, the magnitude of $\nabla T$ cannot be directly measured since no thermometry below the film is possible. The laser induced thermal gradient can, however, be estimated by comparing to a film with a known magneto-thermal coefficient deposited on a similar substrate. For the present study, we use a thin film of the Weyl semimetal $\mathrm{Co}_{2} \mathrm{MnGa}^{33}$. We obtain a thermal gradient of $\nabla T \approx 2 \mathrm{~K} / \mu \mathrm{m}$ for a laser power of $10 \mathrm{~mW}$, as detailed in the Supplementary Note 9 and Supplementary Fig. 7, resulting in an anomalous Nernst coefficient of $1.7 \mu \mathrm{V} / \mathrm{K}$ for the $\mathrm{Mn}_{3} \mathrm{Sn}$ thin film at room temperature. This is higher than the value reported in bulk $\mathrm{Mn}_{3} \mathrm{Sn}$ crystals $0.6 \mu \mathrm{V} / \mathrm{K}^{4}$, which could be due to the higher degree of domain polarization or the more localized detection in our thin film sample. Since the estimation of the thermal gradient contains significant error bars, a more systematic study of the Nernst effect magnitude in thin films is an important future task.

Heat assisted writing. The capability to intentionally write magnetic domains is exceptionally important not only from an application perspective $e^{34}$, but also to quantify spin transfer torque $^{35}$, domain wall motion or giant magnetoresistance ${ }^{36}$ in antiferromagnets. In Fig. 4, we show that magnetic domains can be intentionally written into the $\mathrm{Mn}_{3} \mathrm{Sn}$ film at room temperature, via a combination of high-power $(50 \mathrm{~mW})$ laser illumination and external magnetic fields ${ }^{37,38}$. Hereby, it is of key importance that the external magnetic field does not alter the magnetic texture in $\mathrm{Mn}_{3} \mathrm{Sn}$ at room temperature in the absence of the laser heating (see Fig. 2a, b).

Fig. 4 shows a sequence of writing and erasing of domains at $300 \mathrm{~K}$ using the following procedure: First, the full area (region of interest) is scanned with a $50 \mathrm{~mW}$ laser spot in $+0.5 \mathrm{~T}$ external field applied along $\mathrm{x}$-direction. Subsequently, the same area is investigated using STGM with $10 \mathrm{~mW}$ laser power (Fig. 4a), showing a homogeneous thermo-voltage contrast and, thus, homogeneous $\mathbf{g}$ vector orientation. In the next step, only the area enclosed by the dashed line is scanned with $50 \mathrm{~mW}$, now with applied $-0.5 \mathrm{~T}$, followed by imaging the entire area with STGM with $10 \mathrm{~mW}$ laser power (Fig. 4b). Repeated STGM maps with either $0.5 \mathrm{~T}, 0 \mathrm{~T}$ or $-0.5 \mathrm{~T}$ have no impact on the written pattern (Supplementary Fig. 8), even a magnetic field of $6 \mathrm{~T}$ applied along 


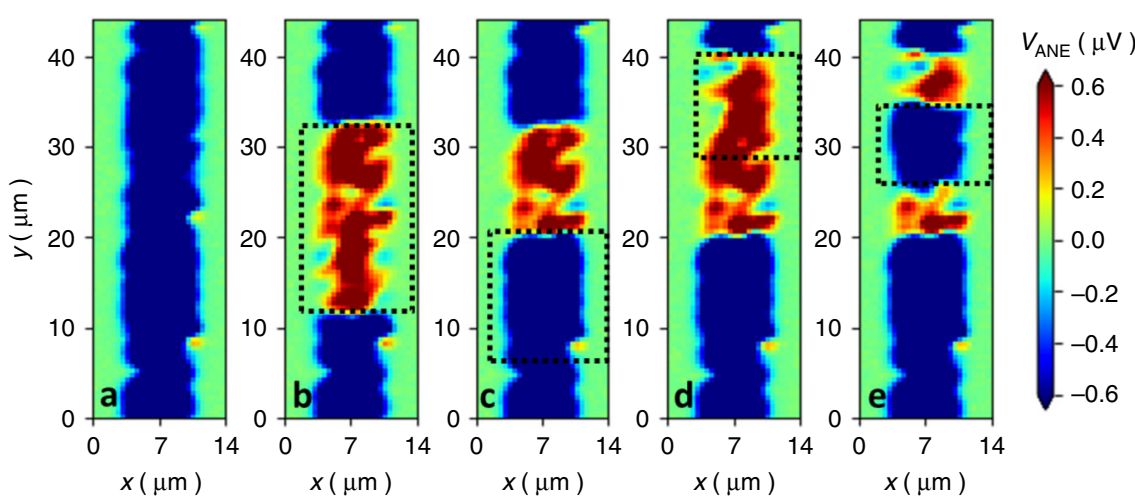

Fig. 4 Domain writing. Sample polarized to single domain (a). In panels $\mathbf{b}-\mathbf{e}$ the area depicted by a dashed line was written using a laser power of $50 \mathrm{~mW}$ and the corresponding polarity of $0.5 \mathrm{~T}$ magnetic field prior to the STGM scan ( $+0.5 \mathrm{~T}$ yields blue contrast and $-0.5 \mathrm{~T}$ yields red contrast). Writing was followed by reading with laser power of $10 \mathrm{~mW}$. The whole sequence was performed at room temperature.

$\mathrm{x}$-direction at $300 \mathrm{~K}$ could not erase the domain pattern (Supplementary Note 10 and Supplementary Fig. 9).

However, increasing the laser power up to $50 \mathrm{~mW}$ again allows us to write domain patterns at will (Fig. $4 c-e)$. In particular, rewriting the same area with the same magnetic field polarity does not alter the $V_{\mathrm{ANE}}$ contrast, while writing with opposite field polarity invariably reverses the sign of the thermo-voltage.

\section{Discussion}

Our work demonstrates that the magnetic structure in the noncollinear antiferromagnet $\mathrm{Mn}_{3} \mathrm{Sn}$ can be spatially mapped out using local laser heating in combination with anomalous Nernst effect measurements. At room temperature and below, the magnetic structure is insensitive to magnetic fields up to $6 \mathrm{~T}$. We further show that domains can be intentionally written into the magnetic structure, via the application of local heat in combination with moderate magnetic fields $( \pm 0.5 \mathrm{~T})$. Our experiments, thus, open a pathway to initializing and detecting a domain pattern in non-collinear antiferromagnetic thin films. This represents a first important step towards studying the intriguing physics of non-collinear AFMs with spatial resolution, such as spin transfer torque-induced domain wall motion or spin flop mechanisms in triangular spin systems. Our technique can be straightforwardly extended to a range of materials, given that they exhibit a finite magneto-thermal response (ANE, anisotropic magneto-thermo power etc.) and, therefore, represents a versatile tool for the investigation of local magnetic properties.

\section{Methods}

Growth. $\mathrm{Mn}_{3} \mathrm{Sn}$ films were grown by a BesTec (https://www.bestec-berlin.de/) UHV magnetron sputtering system on $\mathrm{MgO}$ (111) substrates with a $5 \mathrm{~nm} \mathrm{Ru}$ underlayer. Prior to deposition, the chamber was evacuated to a base pressure of less than $5 \times 10^{-9}$ mbar, while the process gas (Ar $5 \mathrm{~N}$ ) pressure was $3 \times 10^{-3} \mathrm{mbar}$. The $\mathrm{Ru}$ underlayer was deposited at a rate of $0.45 \AA / \mathrm{s}$ by applying $30 \mathrm{~W}$ dc power to a 2 inch target. The $\mathrm{Mn}_{3} \mathrm{Sn}$ films were grown by cosputtering. The $\mathrm{Mn}$ was deposited at a rate of $0.49 \AA / \mathrm{s}$ by applying $42 \mathrm{~W}$ dc power and the $\mathrm{Sn}$ at a rate of $0.30 \AA / \mathrm{s}$ by applying $11 \mathrm{~W}$ dc power to a 3 and 2 inch target, respectively. The growth rates and the film thicknesses were determined by a quartz crystal microbalance and confirmed by using $\mathrm{x}$-ray reflectivity measurements. The substrates were rotated during deposition, to ensure homogeneous growth. The $\mathrm{Ru}$ underlayer was grown at $400^{\circ} \mathrm{C}$, the $\mathrm{Mn}_{3} \mathrm{Sn}$ at RT and finally the stack was postannealed at $300^{\circ} \mathrm{C}$ in situ for $10 \mathrm{~min}$. All films were capped with $3 \mathrm{~nm}$ of Al to prevent oxidation.

Experimental setup. The thermal gradient is generated by a continuous wave laser operating at a wavelength of $\lambda=800 \mathrm{~nm}$ and focused by an objective lens to a spot size of $\sim 1.5 \mu \mathrm{m}$ (see Fig. 1c). The laser power can be continuously tuned by a combination of a half-wave plate and a polarizer. Scanning of the laser spot across the Hall bar is accomplished by moving the objective lens with a $3 \mathrm{D}$ piezopositioner and the thermo-voltage generated along the y-direction is recorded in each position. The laser beam is modulated by a chopper at frequency of $1.7 \mathrm{kHz}$ and the generated thermo-voltage is detected using a lock-in amplifier.

\section{Data availability}

The data that support the findings of this study are available from the corresponding author upon request.

\section{Code availability}

A homemade Python code was used to record and plot the STGM maps and it is available from the corresponding author upon reasonable request. The structural data were analyzed using xrayutilities software (https://xrayutilities.sourceforge.io/)

Received: 29 August 2019; Accepted: 4 November 2019; Published online: 29 November 2019

\section{References}

1. Baltz, V. et al. Antiferromagnetic spintronics. Rev. Mod. Phys 90, 015005 (2018).

2. Jungwirth, T. et al. The multiple directions of antiferromagnetic spintronics. Nat. Phys. 14, 200-203 (2018).

3. Higo, T. et al. Large magneto-optical kerr effect and imaging of magnetic octupole domains in an antiferromagnetic metal. Nat. Photonics 12, 73-78 (2018).

4. Ikhlas, M. et al. Large anomalous nernst effect at room temperature in a chiral antiferromagnet. Nat. Phys. 13, 1085-1090 (2017).

5. Nakatsuji, S., Kiyohara, N. \& Higo, T. Large anomalous hall effect in a noncollinear antiferromagnet at room temperature. Nature 527, 212-215 (2015).

6. Nayak, A. K. et al. Large anomalous hall effect driven by a nonvanishing berry curvature in the noncolinear antiferromagnet $\mathrm{Mn}_{3}$ Ge. Sci. Adv. 2, e1501870 (2016).

7. Suergers, C., Fischer, G., Winkel, P. \& v. Löhneysen, H. Large topological hall effect in the non-collinear phase of an antiferromagnet. Nat. Commun. 5, 3400 (2014).

8. Zhang, W. et al. Spin hall effects in metallic antiferromagnets. Phys. Rev. Lett. 113, 196602 (2014).

9. Kimata, M. et al. Publisher correction: magnetic and magnetic inverse spin hall effects in a non-collinear antiferromagnet. Nature 566, E4-E4 (2019).

10. Kuroda, K. et al. Evidence for magnetic weyl fermions in a correlated metal. Nat. Mater. 16, 1090-1095 (2017).

11. Železný, J., Zhang, Y., Felser, C. \& Yan, B. Spin-polarized current in noncollinear antiferromagnets. Phys. Rev. Lett. 119, 187204 (2017).

12. Gray, I. et al. Spin seebeck imaging of spin-torque switching in antiferromagnetic Pt/NiO heterostructure Phys. Rev. X 9, 041016 (2019).

13. Gray, I. et al. Imaging uncompensated moments and exchange-biased emergent ferromagnetism in ferh thin films. Preprint at https://arxiv.org/abs/ 1906.07243v1 (2019).

14. Weiler, M. et al. Local charge and spin currents in magnetothermal landscapes. Phys. Rev. Lett. 108, 106602 (2012).

15. Kuebler, J. \& Felser, C. Non-collinear antiferromagnets and the anomalous hall effect. EPL (Europhysics Letters) 108, 67001 (2014).

16. Liu, J. \& Balents, L. Anomalous hall effect and topological defects in antiferromagnetic weyl semimetals: $\mathrm{Mn}_{3} \mathrm{Sn} / \mathrm{Ge}$. Phys. Rev. Lett. 119, 087202 (2017). 
17. Manna, K., Sun, Y., Muechler, L., Kübler, J. \& Felser, C. Heusler, weyl and berry. Nat. Rev. Mater. 3, 244-256 (2018).

18. Yang, H. et al. Topological weyl semimetals in the chiral antiferromagnetic materials $\mathrm{Mn}_{3} \mathrm{Ge}$ and $\mathrm{Mn}_{3}$ Sn. New J. Phys. 19, 015008 (2017).

19. Li, X. et al. Chiral domain walls of mn3sn and their memory. Nat. Commun. 10, 3021 (2019).

20. Tomiyoshi, S. \& Yamaguchi, Y. Magnetic structure and weak ferromagnetism of $\mathrm{Mn}_{3} \mathrm{Sn}$ studied by polarized neutron diffraction. J. Phys. Soc. Jpn 51, 2478-2486 (1982).

21. Brown, P. J., Nunez, V., Tasset, F., Forsyth, J. B. \& Radhakrishna, P. Determination of the magnetic structure of $\mathrm{mn} 3 \mathrm{sn}$ using generalized neutron polarization analysis. Journal of Physics: Condensed Matter 2, 9409-9422 (1990).

22. Sticht, J., Hoeck, K.-H. \& Kuebler, J. Non-collinear itinerant magnetism: the case of $\mathrm{Mn}_{3} \mathrm{Sn}$. J. Phys. Condens. Matter 1, 8155-8176 (1989).

23. Sung, N. H., Ronning, F., Thompson, J. D. \& Bauer, E. D. Magnetic phase dependence of the anomalous hall effect in $\mathrm{Mn}_{3} \mathrm{Sn}$ single crystals. Appl. Phys. Lett. 112, 132406 (2018).

24. Zhang, D. et al. First-principles study of the structural stability of cubic, tetragonal and hexagonal phases in $\mathrm{Mn}_{3} \mathrm{Z}(\mathrm{Z}=\mathrm{GA}, \mathrm{Sn}$ and $\mathrm{Ge})$ heusler compounds. J. Phys. Condens. Matter 25, 206006 (2013).

25. Markou, A. et al. Noncollinear antiferromagnetic $\mathrm{Mn}_{3} \mathrm{Sn}$ films. Phys. Rev. Mater. 2, 051001 (2018).

26. Kleiner, W. H. Space-time symmetry of transport coefficients. Phys. Rev. 142, 318-326 (1966).

27. Smejkal, L., Rafael González-Hernández, R., Jungwirth, T. \& Sinova, J. Crystal hall effect in collinear antiferromagnets. Preprint at https://arxiv.org/abs/ 1901.00445 (2019).

28. Guo, G.-Y. \& Wang, T.-C. Large anomalous nernst and spin nernst effects in the noncollinear antiferromagnets $\mathrm{Mn}_{3} \mathrm{X}(\mathrm{X}=\mathrm{Sn}, \mathrm{Ge}, \mathrm{Ga})$. Phys. Rev. B 96, 224415 (2017).

29. Higo, T. et al. Anomalous hall effect in thin films of the weyl antiferromagnet $\mathrm{Mn}_{3}$ Sn. Appl.Physi. Lett. 113, 202402 (2018).

30. You, Y. et al. Anomalous hall effect-like behavior with in-plane magnetic field in noncollinear antiferromagnetic $\mathrm{Mn}_{3} \mathrm{Sn}$ films. Adv. Electron. Mater. 5, 1800818 (2019).

31. Bisson, W. G. \& Wills, A. S. Anisotropy-driven spin glass transition in the kagome antiferromagnet hydronium jarosite, $\left(\mathrm{H}_{3} \mathrm{O}\right) \mathrm{Fe}_{3}\left(\mathrm{SO}_{4}\right)_{2}(\mathrm{OH})_{6}$. J. Phys. Condens. Matter 20, 452204 (2008).

32. Ritchey, I., Chandra, P. \& Coleman, P. Spin folding in the two-dimensional heisenbergkagoméantiferromagnet. Phys. Rev. B 47, 15342-15345 (1993),

33. Reichlova, H. et al. Large anomalous nernst effect in thin films of the weyl semimetal $\mathrm{Co}_{2} \mathrm{MnGa}$. Appl. Phys. Lett. 113, 212405 (2018).

34. Parkin, S. S. P., Hayashi, M. \& Thomas, L. Magnetic domain-wall racetrack memory. Science 320, 190-194 (2008).

35. DuttaGupta, S. et al. Adiabatic spin-transfer-torque-induced domain wall creep in a magnetic metal. Nature Physics 12, 333-336 (2015).

36. Gregg, J. F. et al. Giant magnetoresistive effects in a single element magnetic thin film. Phys. Rev. Lett. 77, 1580-1583 (1996).

37. Mei, A. B. et al. Local photothermal control of phase transitions for ondemand room-temperature rewritable magnetic patterning. Preprint at https://arxiv.org/abs/1906.07239v1 (2019).

38. Singh, U., Echtenkamp, W., Street, M., Binek, C. \& Adenwalla, S. Local writing of exchange biased domains in a heterostructure of co/pd pinned by magnetoelectric chromia. Adv. Funct. Mater. 26, 7470-7478 (2016).

\section{Acknowledgements}

We acknowledge Jakob Lindermeir for technical support, IFW Dresden for providing access to the MST lab, EU FET Open RIA Grant no. 766566, support from the Grant Agency of the Czech Republic under EXPRO grant no. 19-28375X, the Ministry of

Education of the Czech Republic Grants LM2015087 and LNSM-LNSpin, and financial support from the DPG through project B05 and C08 of SFB 1143 (project-id 247310070). D.K. acknowledges the support by the OP Research, Development and Education financed by ESIF and the Czech MSMT (Project No. CZ.02.2.69/0.0/0.0/16_027/0008215). J.Z. acknowledges the Grant Agency of the Czech Republic Grant No. 19-18623Y and support from the Institute of Physics of the Czech Academy of Sciences and the Max Planck Society through the Max Planck Partner Group programme.

\section{Author contributions}

H.R., J.W. and S.T.B.G planned and designed experiments. A.M., D.K., Z.S. and C.F. provided material and characterization. H.R., T.Ja., J.G., R.S., D.K. M.B., H.S., P.N. and J.W. built the experimental setup, performed and analyzed the experiments. H.R., S.T.B. G, J.W., J.Z. and T.Ju. interpreted the data. H.R. and S.T.B.G. wrote the paper with contributions from all authors.

\section{Competing interests}

The authors declare no competing interests.

\section{Additional information}

Supplementary information is available for this paper at https://doi.org/10.1038/s41467019-13391-z.

Correspondence and requests for materials should be addressed to H.R.

Peer review information Nature Communications thanks the anonymous reviewers for their contribution to the peer review of this work. Peer reviewer reports are available.

Reprints and permission information is available at http://www.nature.com/reprints

Publisher's note Springer Nature remains neutral with regard to jurisdictional claims in published maps and institutional affiliations.

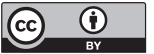

Open Access This article is licensed under a Creative Commons Attribution 4.0 International License, which permits use, sharing, adaptation, distribution and reproduction in any medium or format, as long as you give appropriate credit to the original author(s) and the source, provide a link to the Creative Commons license, and indicate if changes were made. The images or other third party material in this article are included in the article's Creative Commons license, unless indicated otherwise in a credit line to the material. If material is not included in the article's Creative Commons license and your intended use is not permitted by statutory regulation or exceeds the permitted use, you will need to obtain permission directly from the copyright holder. To view a copy of this license, visit http://creativecommons.org/ licenses/by/4.0/.

(C) The Author(s) 2019 